\title{
Needling and open filtering bleb revision after XEN-45 implantation-a retrospective outcome comparison
}

\author{
Stefan Steiner ${ }^{1} \cdot$ Hemma Resch $^{1} \cdot$ Barbara Kiss $^{1} \cdot$ Daniel Buda $^{1} \cdot$ Clemens Vass $^{1}$ (D)
}

Received: 12 February 2021 / Revised: 26 March 2021 / Accepted: 19 April 2021 / Published online: 11 May 2021

(c) The Author(s) 2021

\begin{abstract}
Purpose To compare efficacy and safety of needling and open bleb revision after XEN-45 surgery.

Methods This retrospective study represents real-life data of patients who underwent XEN-45 surgery between November 2014 and June 2018 in the Vienna General Hospital. The following groups were formed for data evaluation: (PSEA) primary surgery secondary intervention allowed $(n=268)$; (PS) primary surgery until secondary intervention $(n=268)$; (N) first needling until additional secondary intervention $(n=55)$; (BR) first bleb revision until additional secondary intervention $(n=105)$. Main outcome measures were pre- and postoperative intraocular pressure (IOP), number of glaucoma medication (GM), Kaplan-Meier success rates, and secondary intervention rates. Success was defined as postoperative IOP $<21 \mathrm{mmHg}$ and $<18 \mathrm{mmHg}$ together with $\geq 20 \%$ IOP reduction with medication allowed.

Results IOP (and GM) was lowered from $23.5 \pm 8.0$ (GM 3.1 \pm 1.0 ) to $14.9 \pm 8.2 \mathrm{mmHg}(1.2 \pm 1.4$ ) in group PSEA and $18.1 \pm 8.2 \mathrm{mmHg}(1.5 \pm 1.4)$ in group PS, in group $\mathrm{N}$ from $23.2 \pm 10.1(1.5 \pm 1.0)$ to $19.3 \pm 8.5 \mathrm{mmHg}(2.2 \pm 1.3)$ and in group BR from $22.0 \pm 8.0 \mathrm{mmHg}(2.5 \pm 1.1)$ to $15.5 \pm 6.4 \mathrm{mmHg}(1.3 \pm 1.5)$ after a median follow-up of 16.0, 8.4, 4.8, and 7.3 months, respectively. Success rates at 1 year were significantly higher in group BR (50.7\%) compared to PS (37.7\%, $p=0.019)$ and $\mathrm{N}(24.3 \% ; p=0.015)$. An additional intervention was required less frequently in group BR $(17.1 \%)$ compared to group PS $(49.6 \%, p<0.001)$ and group $\mathrm{N}(54.5 \%, p<0.001)$.

Conclusion Our data appear to indicate favorable outcomes for open XEN bleb revision in terms of Kaplan-Meier success rates and secondary intervention rate compared to the needling procedure.
\end{abstract}

Keywords XEN $\cdot$ Needling $\cdot$ Bleb revision $\cdot$ MIGS $\cdot$ Glaucoma surgery $\cdot$ Filtering surgery

\section{Key messages}

- The XEN-45 procedure is a safe and effective minimally invasive glaucoma surgery

- The XEN-45 surgery is followed by extensive postoperative care including a high rate of secondary interventions like needling or open filtering bleb revision

- Open filtering bleb revision might have favorable outcomes in terms of intraocular pressure control, number of glaucoma medication, success rate, and secondary intervention rate compared to the needling procedure

Clemens Vass

clemens.vass@meduniwien.ac.at

1 Department of Ophthalmology and Optometry, Medical University of Vienna, Währinger Gürtel 18-20, 1090 Vienna, Austria

\section{Introduction}

Trabeculectomy is the most commonly performed glaucoma surgery with a good intraocular pressure (IOP) lowering effect [1]. Nevertheless, many types of minimally invasive 
glaucoma surgery (MIGS) like iStent [2-4] (Glaukos Corporation, Laguna Hills, CA), trabectome [3, 5] (NeoMedix, Tustin, CA), canaloplasty [6], CyPass [7] (Alcon, Fort Worth, TX), Hydrus [4] (Ivantis, Inc, Irvine, CA), and Kahook dual blade [8] (New World Medical Inc., Rancho Cuamonga, CA) were introduced to provide an improved safety profile, similar IOP reduction compared to trabeculectomy, and less postoperative care and interventions. These new methods have in common that IOP regulation is not as good as with a traditional trabeculectomy even though the safety profile is preferable in MIGS [9].

The XEN-45 (Allergan Inc., Irvine, CA) implant by Allergan is a glaucoma drainage device aiming for wellcontrolled long-term IOP reduction and a good safety profile with uncomplicated postoperative patient management. Current literature shows that satisfying postoperative IOP levels can be reached but at the expense of very frequent bleb needlings or surgical revisions [10-16]. Despite some evidence on the effectiveness of needling [17] and open filtering bleb revision [18] after XEN-45, data about the XEN-45 surgery itself, without needling or revision, as well as comparative data about the effectiveness of bleb revision vs. needling are still missing.

\section{Materials and methods}

In this retrospective study, we included all patients who underwent XEN-45 gel stent implantation at the Vienna General Hospital in the period from November 2014 to June 2018. No exclusion criteria were defined. The study was approved by the local ethics committee and followed the tenets of the Declaration of Helsinki.

The surgical technique of XEN-45 was the usual ab interno implantation following a preoperative subconjunctival injection of 0.05 to $0.1 \mathrm{ml}$ of either $0.1 \mathrm{mg} / \mathrm{ml}$ or $0.2 \mathrm{mg} /$ $\mathrm{ml}$ Mitomycin C (MMC) solution. Open bleb revision was performed in a similar way as published recently by Linton [18]. First, a six- to 9-mm wide conjunctival peritomy was performed. To have enough access, we usually supplemented this with a nasally located radial conjunctival incision. As soon as the implant was located, scar tissue was carefully removed around the external tip of the XEN-45. In the event that no flow was achieved, the device was intubated with a $10-0$ or 9-0 nylon suture $(n=5)$, or if unsuccessful $(n=1)$, or in case of inadvertent damage to the XEN-45, the eye was given a new shunt $(n=15)$. Eyes that received a new shunt were also included in our analysis. In most cases, tenonectomy was performed in an area approximately one clock hour position to both sides of the XEN-45 and as far posterior as achievable. At the end of the procedure, the conjunctiva was closed with $8-0$ vicryl sutures, and we subconjunctivally injected $0.1 \mathrm{ml}$ of $0.2 \mathrm{mg} / \mathrm{ml} \mathrm{MMC} \mathrm{solution.}$
Bleb needling was done at the slit lamp using a $27 \mathrm{G}$ needle or the bent needling knife (Kai Industries Co., Ltd., Oyana, Japan), followed by subconjunctival injection of $0.1 \mathrm{ml}$ of $0.2 \mathrm{mg} / \mathrm{ml} \mathrm{MMC}$ solution.

Main outcome measures were pre- and postoperative IOP, pre- and postoperative number of glaucoma medication (GM), complications (hypotony, reduction of anterior chamber depth, choroidal detachments, maculopathy, hyphema, infection), secondary interventions (defined as needling or open filtering bleb revision), and other secondary glaucoma surgery. Hypotony was defined as IOP $<6 \mathrm{mmHg}$ at any single measurement and reduction of anterior chamber depth as peripheral iridocorneal touch or worse. Additionally, we report the Kaplan-Meier success rates for the different groups and success criteria.

Four groups were formed for data evaluation: (1) primary surgery with everything allowed (PSEA); (2) primary surgery (PS); (3) needling (N); (4) bleb revision (BR). All eyes were included in the PSEA group, where any number of secondary interventions was allowed, but any other secondary glaucoma surgery was considered a failure. The PS group also comprised all eyes, but for this group as well as for the $\mathrm{N}$ and BR group, any secondary intervention or secondary glaucoma surgery was deemed a failure. Any first needling after primary surgery was included in the $\mathrm{N}$ group, and similarly, any first bleb revision was included in the BR group. Included groups are shown in Fig. 1.

Patient data were retrieved retrospectively from patient files and were categorized in the following time periods: day $1-2$, day $3-7$, day $8-14$, day $15-30$, month $>1-2$, month $>2-6$, month $>6-12$, month $>12-24$, month $>24-36$, month $>36-48$, and month $>48$ until last follow-up. Up to three most recent preoperative data records that determined the surgical indication were considered for data analysis individually for the PSEA/PS, N, and the BR group.

Qualified success was defined as IOP below $21 \mathrm{mmHg}$ and below $18 \mathrm{mmHg}$ together with an IOP reduction of $20 \%$ or more (QS21 and QS18) and glaucoma medication allowed. Another success criterion was $20 \%$ or more IOP reduction or reduction in the number of Glaucoma Medication of 2 or more (IoGM). This was done to reflect the praxis that in some patients, the main goal of the XEN-45 was to reduce glaucoma medication.

Kaplan-Meier survival analysis was carried out to compare failure rates of the PS, N, and BR group with the success criteria QS21, QS18, and IoGM. Failure was determined as not meeting the mentioned criteria on two consecutive visits after 60 days of follow-up or at the last visit.

Subgroup analysis was performed to evaluate success in eyes with a preoperative IOP of $\leq 21 \mathrm{mmHg}$ and a preoperative IOP of $>21 \mathrm{mmHg}$ and to analyze differences in success between stand-alone XEN-45 implantation and combined XEN-45 surgery with cataract extraction and intraocular lens implantation. 
Fig. 1 Additional interventions following Xen primary surgery; number of patients, median interval in months, in parenthesis $=$ interquartile range; *included and analyzed groups

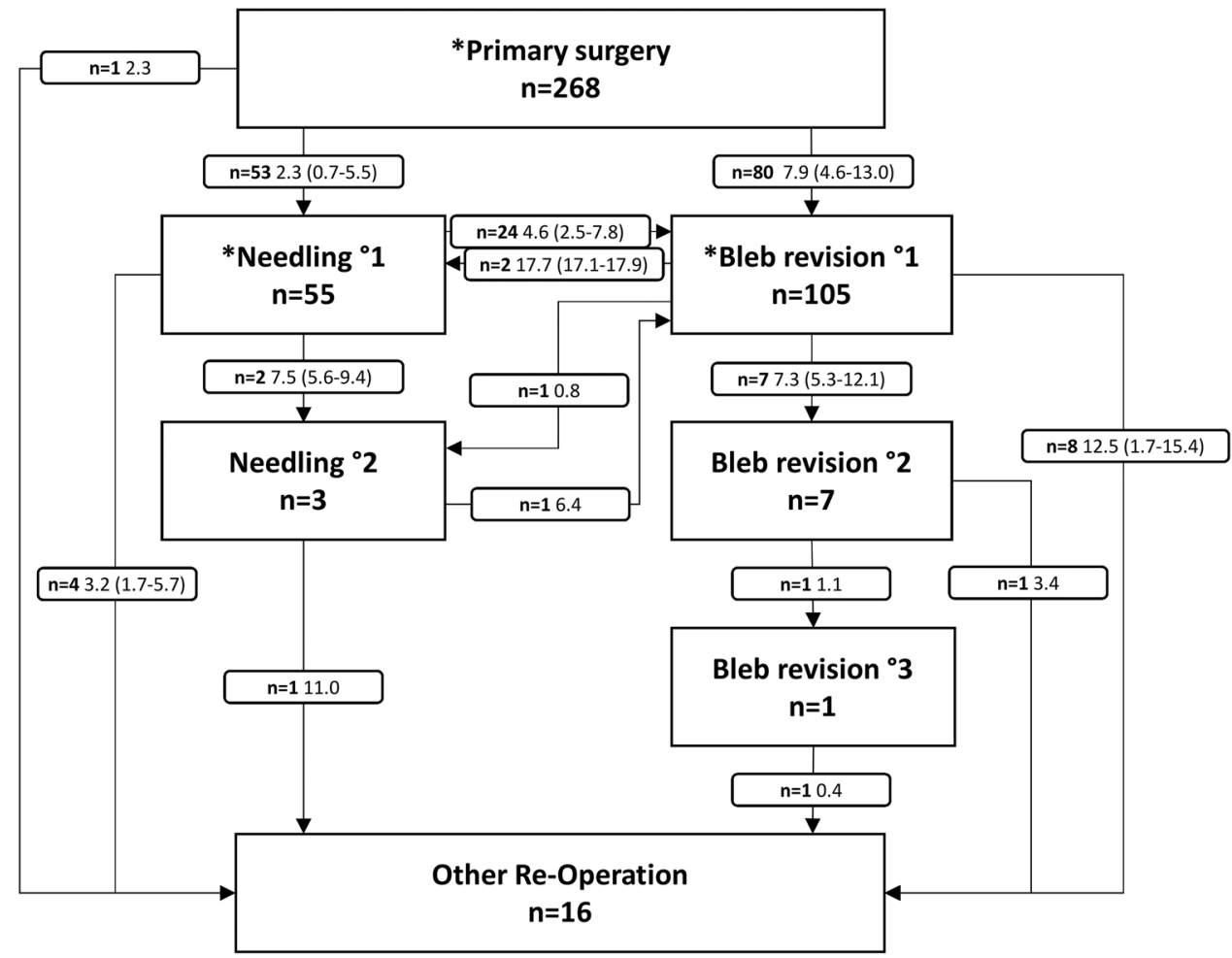

Statistical analyses were performed using SPSS $®$ Version 21. Paired $t$ test was used to compare pre- and postoperative IOP and GM changes. The unpaired $t$ test was used to compare pre- and postoperative IOP and GM between our defined groups at certain time periods. Chi-square test was used to calculate differences in the occurrence of additional interventions and complications between groups. Success rates in our Kaplan-Meier analyses were compared using the Log Rank test pairwise over strata. Multivariate cox proportional hazards models were calculated to stratify baseline risk factors for failure in the PS group and the BR vs. N group. All tests were two-sided with $p$ values $<0.05$ considered as statistically significant.

\section{Results}

The baseline characteristics of the study groups are shown in Table 1. A total of 268 eyes of 222 patients who underwent XEN-45 surgery were included in our analysis. The median age of the whole cohort was $68.2(54.0-76.6)$ years. The ratio between right and left eyes was 142 to 126 , and the male to female ratio was 130 to 138 . The XEN-45 surgery was performed as a stand-alone procedure in 193 eyes and as combined procedure with cataract extraction in 75 eyes.

Baseline IOP in group PSEA/PS was $23.5 \mathrm{mmHg}$ and was lowered to $14.9 \mathrm{mmHg}$ in the PSEA group and to
18.1 $\mathrm{mmHg}$ in the PS group after a median follow-up of 16.0 vs. 8.4 months, respectively. The number of GM was reduced from 3.1 to 1.2 in group PSEA and 1.5 in group PS. In group $\mathrm{N}$ and group BR, the preoperative IOP was 23.2 vs. $22.0 \mathrm{mmHg}$ and was lowered to $19.3 \mathrm{vs} .15 .5 \mathrm{mmHg}$. GM changed from 1.5 vs. 2.5 to 2.2 vs. 1.3 after a median follow-up period of 4.8 vs. 7.3 months in the $\mathrm{N}$ vs. BR group, respectively. The time course of IOP and GM is shown in Fig. 2. No significant differences in baseline IOP were observed between group PS, N, and BR. Baseline GM was significantly higher in group PS compared to group $\mathrm{N}$ $(p<0.001)$ and BR $(p<0.001)$. GM at baseline was also higher in group BR than in group $\mathrm{N}(p<0.001)$. From the immediate postoperative period throughout the first 2 years, IOP and GM were very similar between PSEA, PS, and BR. Contrary to this, the $\mathrm{N}$ group had significantly higher IOP during the first 2 months, followed by a reduction to the same level compared to all other groups after the second month.

Following XEN-45 implantation, in 53 eyes, the first secondary intervention was needling, whereas it was bleb revision in 80 eyes. While 25 eyes (47\%) of the former needed subsequent bleb revision, only two eyes $(2.5 \%)$ of the latter required subsequent needling. These eyes, receiving their first needling after previous bleb revision or vice versa, were also included in either the $\mathrm{N}$ group or BR group, which made a total of 55 eyes in the $\mathrm{N}$ group and 105 eyes in the BR group. 
Table 1 Baseline characteristics

\begin{tabular}{|c|c|c|c|}
\hline & $\begin{array}{l}\text { Primary surgery } \\
n=268\end{array}$ & $\begin{array}{l}\text { Needling } \\
n=55\end{array}$ & $\begin{array}{l}\text { Bleb revision } \\
n=105\end{array}$ \\
\hline Age (years), median (IQR) & $68.2(54.0-76.6)$ & $67.8(52.1-77.0)$ & $68.48(54.1-76.8)$ \\
\hline \multicolumn{4}{|l|}{ Race, $n(\%)$} \\
\hline Caucasian & $264(99)$ & $55(100)$ & $103(98)$ \\
\hline Asian & $4(1)$ & $0(0)$ & $2(2)$ \\
\hline \multicolumn{4}{|l|}{ Gender, $n(\%)$} \\
\hline Female & $130(49)$ & $27(49)$ & $52(49)$ \\
\hline Male & $138(51)$ & $28(51)$ & $53(51)$ \\
\hline \multicolumn{4}{|l|}{$\mathrm{IOP}(\mathrm{mm} \mathrm{Hg})$ and $\mathrm{GM}$, mean $\pm \mathrm{SD}$} \\
\hline IOP baseline & $23.5 \pm 8.0$ & $23.2 \pm 10.1$ & $22 \pm 8.0$ \\
\hline GM baseline & $3.1 \pm 1.0$ & $1.5 \pm 1.4$ & $2.5 \pm 1.1$ \\
\hline \multicolumn{4}{|l|}{ Diagnosis, $n(\%)$} \\
\hline Primary open angle & $79(29)$ & $16(29)$ & $30(29)$ \\
\hline Primary open angle (normal tension) & $16(6)$ & $2(4)$ & $6(6)$ \\
\hline Pseudoexfoliative & $60(22)$ & $13(24)$ & $22(21)$ \\
\hline Pigment dispersion & $16(6)$ & $4(7)$ & $7(7)$ \\
\hline Primary angle closure & $29(11)$ & $6(11)$ & $12(11)$ \\
\hline Juvenile open angle & $21(8)$ & $2(4)$ & $6(6)$ \\
\hline Secondary glaucoma* & $22(8)$ & $5(9)$ & $8(8)$ \\
\hline Ocular hypertension & $20(7)$ & $6(11)$ & $10(10)$ \\
\hline Congenital glaucoma & $5(2)$ & $1(2)$ & $4(4)$ \\
\hline \multicolumn{4}{|l|}{ Previous eye surgery or laser, $n(\%)$} \\
\hline Posterior intraocular lens & $83(31)$ & $13(24)$ & $34(32)$ \\
\hline Trabeculectomy & $27(10)$ & $6(11)$ & $10(10)$ \\
\hline Trabeculotomy & $7(3)$ & $1(2)$ & $4(4)$ \\
\hline Baerveldt & $3(1)$ & $1(2)$ & $1(1)$ \\
\hline Ahmed & $2(1)$ & $0(0)$ & $1(1)$ \\
\hline iStent & $4(1)$ & $0(0)$ & $1(1)$ \\
\hline Hydrus & $1(0)$ & $1(2)$ & $0(0)$ \\
\hline Kahook & $2(1)$ & $0(0)$ & $0(1)$ \\
\hline Cyclodestructive procedure & $5(2)$ & $2(4)$ & $3(3)$ \\
\hline Laser peripheral iridotomy & $26(10)$ & $8(15)$ & $10(10)$ \\
\hline Laser trabeculoplasty & $10(4)$ & $2(4)$ & $5(5)$ \\
\hline Laser iridoplasty & $5(2)$ & $0(0)$ & $3(3)$ \\
\hline
\end{tabular}

$I Q R$ interquartile range

*Secondary glaucoma excluding pseudoexfoliative and pigment dispersion glaucoma

All secondary interventions after XEN-45 primary surgery with median intervals are shown in the flow chart in Fig. 1. Three out of 55 eyes in the $\mathrm{N}$ group needed a second needling, 25 eyes a bleb revision, and five eyes required any other glaucoma surgery, whereas in the BR group, seven out of 105 eyes needed a second and one eye a third bleb revision, three eyes required needling, and eight eyes any other glaucoma surgery. In total, other glaucoma surgeries were necessary in 16 eyes (ten trabeculectomies, one re-trabeculectomy, three Ahmed valve implants, one Baerveldt implant, and one cyclodestructive procedure). The cyclodestructive procedure was performed after XEN-45 primary surgery without any needling or bleb revision in a patient after retinal detachment surgery. An additional IOP lowering secondary intervention or other secondary glaucoma surgery was required less frequently in Group BR $(n=18 / 105,17.1 \%)$ compared to group PS $(n=133 / 268,49.6 \%, p<0.001)$ and group $\mathrm{N}(n=30 / 55,54.5 \%, p<0.001)$, while no significant difference was observed between group PS and $\mathrm{N}$.

\section{Success rates}

Kaplan-Meier curves of the PS, N, and BR group for the QS21 and IoGM criteria are shown in Fig. 3a. 


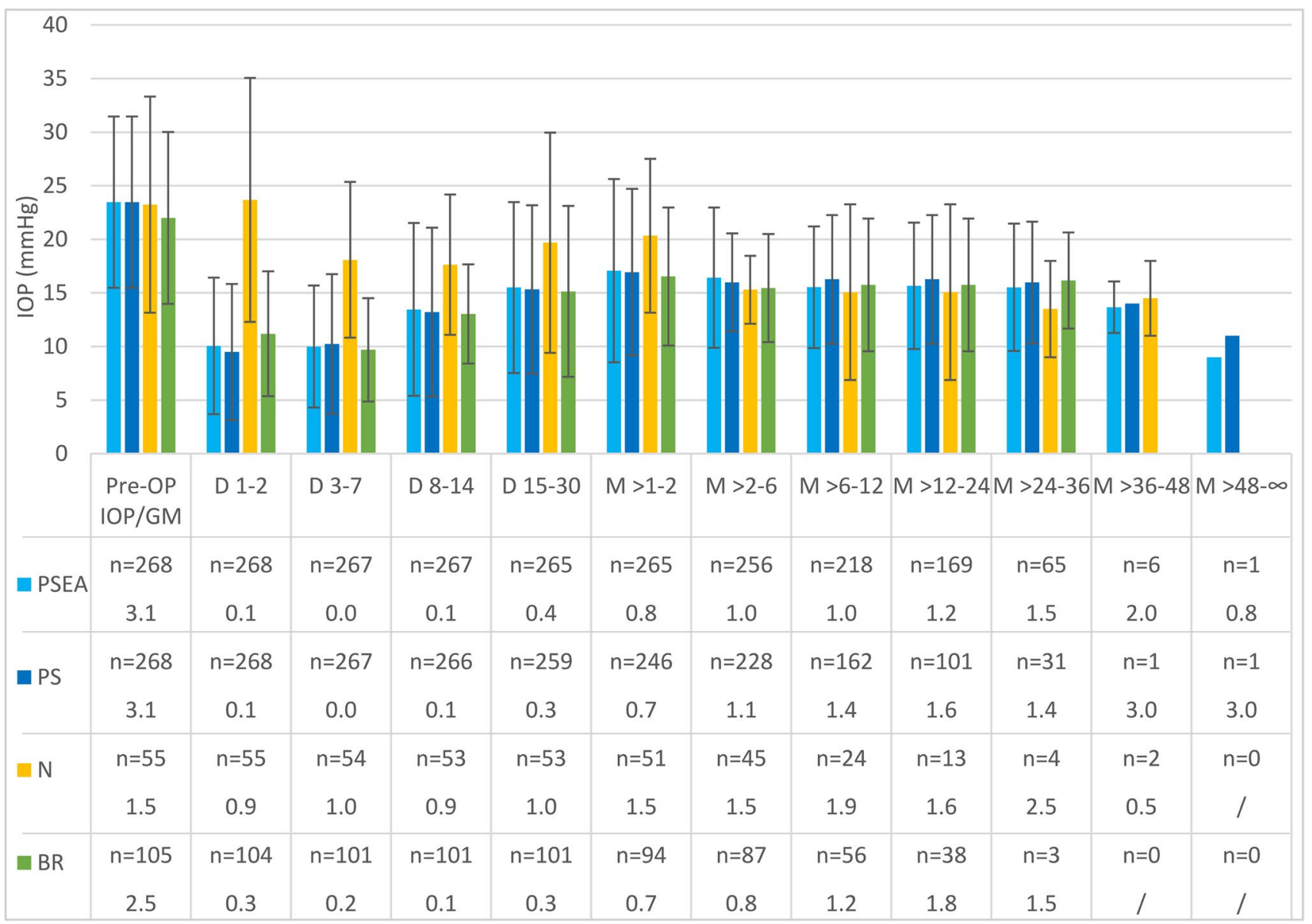

Fig. 2 IOP and glaucoma medication over time in the groups PSEA (primary surgery everything allowed), PS (primary surgery), N (needling), BR (bleb revision), D (day), and M (month)

Kaplan-Meier success rates (and standard error) for the QS21 criteria at 1 year were $37.7 \%$ (3.4\%), $24.3 \%$ (7.8\%), and $50.7 \%$ (6.1\%) for the PS, N, and BR group, respectively. For the stricter QS18 criteria, the success rates were lower, and for the IoGM criteria, the success rates were higher compared to the QS21 criteria. For both the QS21 and the QS18 criteria, survival was significantly better in the BR group compared to the PS (log rank, QS21 $p=0.019$; QS18 $p=0.016)$ and $\mathrm{N}$ group ( $\log$ rank QS21 $p=0.015$; QS18 $p=0.016$ ), while there was no difference between the PS and N group. Survival for the IoGM criteria was lower in group $\mathrm{N}$ compared to $\mathrm{BR}(\log$ rank $p=0.003)$ and PS (log rank $p=0.039)$ and there was no difference between group PS and BR.

The Kaplan-Meier curves of the subgroups with a preoperative IOP of $>21 \mathrm{mmHg}$ are shown in Fig. $3 \mathrm{~b}$ and with preoperative IOP of $\leq 21 \mathrm{mmHg}$ in Fig. 3c. Patients with a preoperative IOP of $>21 \mathrm{mmHg}$ showed better survival rates at one year in the PS group (log rank QS21 $p<0.001$; QS18 $p<0.001$; IoGM $p<0.001$ ) and in the BR group (log rank QS21 $p=0.002$; QS18 $p<0.001$, IoGM n.s.) compared to the subgroup with IOP $\leq 21 \mathrm{mmHg}$. No differences were observed in the $\mathrm{N}$ group for the QS21, QS18, and IoGM criteria. Regarding the subgroup with IOP $\leq 21 \mathrm{mmHg}$, the success criterion IoGM yielded better survival compared to the QS21 or the QS18 criteria. The latter two were virtually identical. Again, the survival was better for the BR group compared to both other groups.

The combined XEN-45 primary surgery with cataract extraction showed a tendency towards better Kaplan-Meier survival rates compared to the stand-alone procedure in the QS21 and QS18 criteria. Nevertheless, this difference was not statistically significant. Also, no significant differences were observed between eyes with prior stand-alone and combined primary surgeries in the $\mathrm{N}$ and $\mathrm{BR}$ group for the QS21, QS18, and IoGM criteria (Table 2).

Hazard ratios calculated with the use of Cox proportional hazards models are shown in Table 3 . In the model of the PS group, higher baseline IOP (HR $=0.94$ per $\mathrm{mmHg}, p<0.001)$ and a combined XEN-45 surgery with cataract extraction $(\mathrm{HR}=0.55, p=0.01)$ lowered the risk 


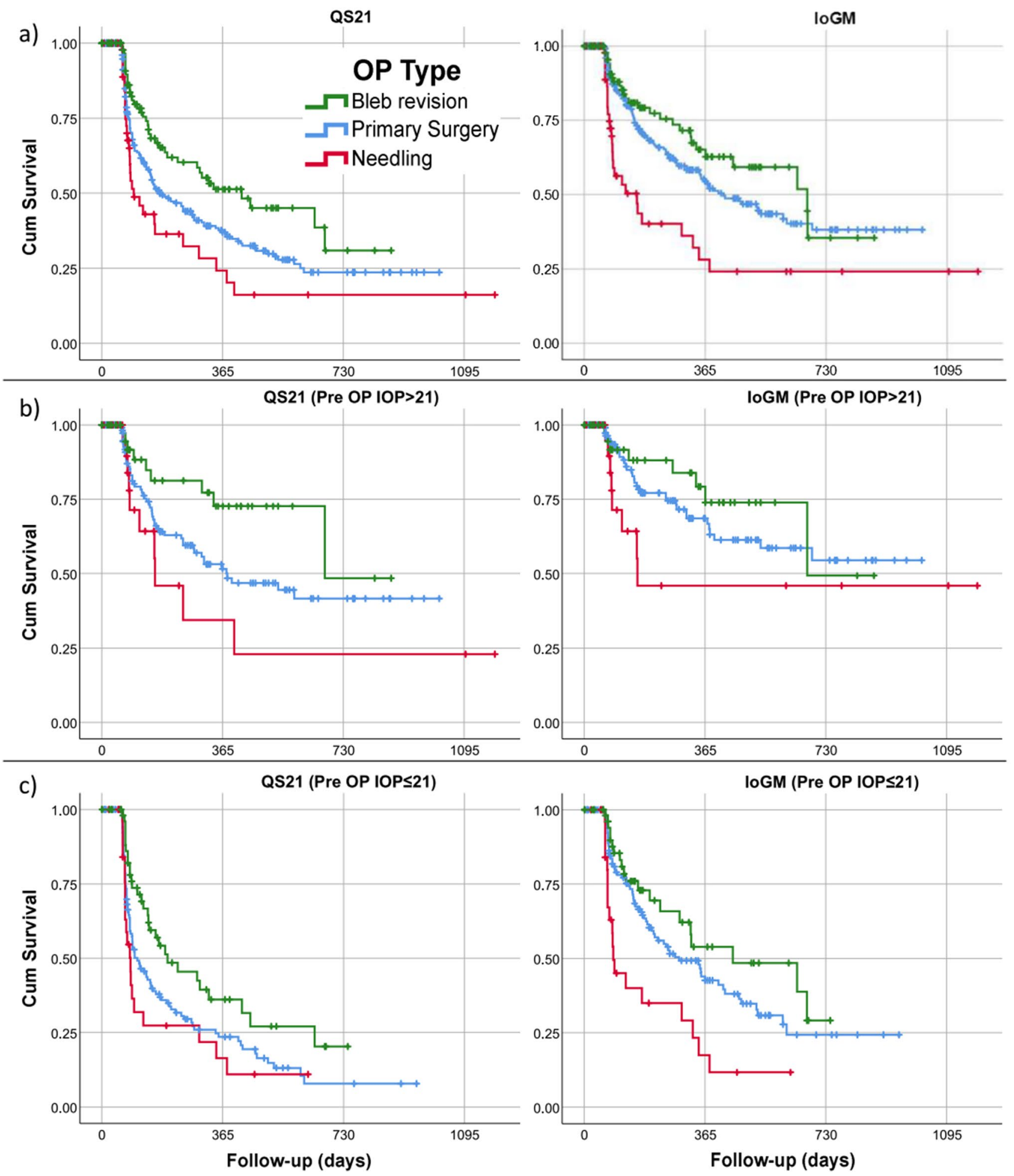

Fig. 3 Kaplan-Meier plots comparing success rates of groups PS (primary surgery), $\mathrm{N}$ (needling), and BR (bleb revision) according to

a preoperative IOP of $>21 \mathrm{mmHg}$, and (c) in the subgroup with a preoperative IOP of $\leq 21 \mathrm{mmHg}$, IoGM ( $20 \%$ or more IOP reduction or reduction in the number of glaucoma medication of 2 or more) 
Table 2 Median survival time and standard error (SE) in days for the primary surgery (PS), needling $(\mathrm{N})$, and bleb revision (BR) group and for the subgroups stand-alone vs. combined primary XEN-45 implantation with cataract extraction using the QS21, QS18, and IoGM success criteria*

\begin{tabular}{|c|c|c|c|c|c|c|}
\hline & \multicolumn{2}{|l|}{ Overall } & \multicolumn{2}{|c|}{ Stand-alone } & \multicolumn{2}{|c|}{ Combined } \\
\hline & \multicolumn{2}{|c|}{ Median survival } & \multicolumn{2}{|c|}{ Median survival } & \multicolumn{2}{|c|}{ Median survival } \\
\hline & Estimate & SE & Estimate & SE & Estimate & SE \\
\hline Primary surgery & $n=268$ & & $n=193$ & & $n=75$ & \\
\hline QS21 & 176 & 31 & 155 & 14 & 278 & 47 \\
\hline QS18 & 151 & 21 & 148 & 9 & 247 & 47 \\
\hline IoGM & 420 & 63 & 405 & 72 & 425 & 105 \\
\hline Needling & $n=55$ & & $n=38$ & & $n=17$ & \\
\hline QS21 & 97 & 22 & 87 & 18 & 125 & 50 \\
\hline QS18 & 87 & 8 & 86 & 5 & 97 & 31 \\
\hline IoGM & 159 & 45 & 114 & 54 & 161 & 119 \\
\hline Bleb revision & $n=105$ & & $n=82$ & & $n=23$ & \\
\hline QS21 & 423 & 134 & 423 & 156 & 449 & 93 \\
\hline QS18 & 323 & 101 & 338 & 138 & 323 & 62 \\
\hline IoGM & 673 & 158 & 673 & 18 & 449 & 116 \\
\hline
\end{tabular}

SE, standard error

*QS21 and QS18, postoperative IOP $<21 \mathrm{mmHg}$ and $<18 \mathrm{mmHg}$ together with $\geq 20 \%$ IOP reduction; IoGM, $20 \%$ or more IOP reduction or reduction in the number of glaucoma medication of 2 or more of failure. All other covariates were not statistically significant. No interactions between type of glaucoma and combined or stand-alone procedures were observed in a sub-model.

In the $\mathrm{N}$ vs. BR group model, BR as revision type was strongly associated with a lower risk of failure regarding the QS21 criteria $(\mathrm{HR}=0.38, p<0.001)$. Higher baseline IOP was a protective factor $(\mathrm{HR}=0.89$ per $\mathrm{mmHg}$, $p<0.001)$. Male gender was a risk factor for failure
$(\mathrm{HR}=1.99, p=0.02)$. All other putative factors, including the interval to primary surgery, were not statistically significant.

\section{Complications}

All three procedures, the XEN-45 implantation, the needling, and the bleb revision were followed by a small number of complications (Table 4). Transient ocular hypotony

Table 3 Hazard ratios for not meeting the QS21 criterium within the primary surgery (PS) group and needling (N) vs. bleb revision (BR) groups*

\begin{tabular}{|c|c|c|c|c|}
\hline \multirow[b]{2}{*}{ Covariates } & \multicolumn{2}{|l|}{ Primary surgery } & \multicolumn{2}{|c|}{ Needlings and bleb revisions } \\
\hline & Hazard ratio $(95 \% \mathrm{CI})$ & $P$ value & Hazard ratio $(95 \% \mathrm{CI})$ & $P$ value \\
\hline Sex: male & $0.85(0.60-1.20)$ & 0.35 & $1.99(1.19-3.35)$ & 0.01 \\
\hline Age & $1.00(0.99-1.01)$ & 0.95 & $1.00(0.97-1.02)$ & 0.66 \\
\hline IOP baseline & $0.94(0.91-0.97)$ & $<0.001$ & $0.89(0.85-0.94)$ & $<0.001$ \\
\hline GM baseline & $0.94(0.78-1.15)$ & 0.56 & $0.99(0.78-1.24)$ & 0.90 \\
\hline Diagnosis: primary open angle ${ }^{1}$ & 1.00 & 0.50 & 1.00 & 0.62 \\
\hline Diagnosis: PEX or pigmentary ${ }^{1}$ & $0.72(0.47-1.11)$ & 0.13 & $0.84(0.46-1.55)$ & 0.59 \\
\hline Diagnosis: angle closure ${ }^{1}$ & $0.99(0.55-1.78)$ & 0.96 & $0.59(0.24-1.44)$ & 0.25 \\
\hline Diagnosis: other ${ }^{1}$ & $0.91(0.51-1.64)$ & 0.76 & $0.65(0.28-1.50)$ & 0.31 \\
\hline Combined XEN with cataract extraction & $0.55(0.36-0.85)$ & 0.01 & & \\
\hline Conjunctival opening surgery prior to XEN & $0.82(0.56-1.20)$ & 0.31 & & \\
\hline Revision type: bleb revision & & & $0.38(0.21-0.70)$ & $<0.001$ \\
\hline Needling prior to bleb revision & & & $1.20(0.55-2.59)$ & 0.65 \\
\hline Bleb revision prior to needling & & & $2.35(0.40-13.95)$ & 0.35 \\
\hline Interval to primary surgery & & & $1.00(1.00-1.00)$ & 0.75 \\
\hline
\end{tabular}

*Hazard ratios calculated using Cox proportional hazards models

${ }^{1}$ Primary open angle was defined as the reference diagnosis 
Table 4 Complications after primary surgery (PS), needling (N), and bleb revision (BR)

\begin{tabular}{llll}
\hline & $\begin{array}{l}\text { Primary surgery } \\
n=268\end{array}$ & $\begin{array}{l}\text { Needling } \\
n=55\end{array}$ & $\begin{array}{l}\text { Bleb revision } \\
n=105\end{array}$ \\
\hline $\begin{array}{c}\text { Complications, } n(\%) \\
\text { Transient hypotony }\end{array}$ & $81(30)^{* * *}$ & $3(6)$ & $18(17)$ \\
$\quad \begin{array}{l}\text { Shallow anterior } \\
\text { chamber }\end{array}$ & $11(4)^{*}$ & $0(0)$ & $0(0)$ \\
$\quad \begin{array}{l}\text { Choroidal detach- } \\
\text { ment/folds }\end{array}$ & $38(14)^{* *}$ & $0(0)$ & $0(0)$ \\
$\quad \begin{array}{l}\text { Bleb leak } \\
\text { Corneal dellen }\end{array}$ & $5(2)$ & $1(2)$ & $4(4)$ \\
Any complication per & $95(35)^{* * *}$ & $0(0)$ & $2(2)$ \\
eye & & $4(7)$ & $24(23)$ \\
\hline
\end{tabular}

${ }^{\S}$ Shallow anterior chamber defined as peripheral iridocorneal touch or worse; ( $<<0.05$ comparing * PS vs. BR, ** PS vs. BR and $\mathrm{N}, * * *$ difference between all groups)

occurred in $30.2 \%$ after PS, $17.1 \%$ after BR, and $5.5 \%$ after $\mathrm{N}$ with statistically significant differences $(p<0.05)$ between all groups. The average duration of ocular hypotony was 12 days after PS, 7 days after N, and 7 days after BR. The time of hypotony exceeded 30 days in 4 patients of the PS group and lasted 30 or fewer days in all cases of the $\mathrm{N}$ and BR group. There was no case with persistent ocular hypotony. Shallow anterior chamber was significantly more frequent in PS (4.1\%) than in N (0\%) and BR (0\%). Choroidal detachments or folds were significantly more frequently observed after PS (14.2\%) compared to N $(0.0 \%)$ and BR $(0.0 \%)$. The average duration of choroidal detachments or folds in the PS group was 28 days, whereas 5 choroidals lasted longer than 30 days. Kissing choroidal detachments or choroidals with macular involvement occurred in 5 eyes after PS only. All patients who suffered choroidal detachments received atropine eye drops ( $1 \%$ or $2 \%$ ) once daily until complete spontaneous recovery, while sometimes this was supported by anterior chamber reformation $(n=5)$. One kissing choroidal detachment originated from a delayed choroidal hemorrhage which was surgically drained. In $35.4 \%$ of the patients after PS, at least one of the mentioned complications occurred. For the N and BR group, that holds true for $7.2 \%$ and $22.9 \%$ of the patients. These differences were statistically significant between all groups (PS vs. N $p<0.001$, PS vs. $\operatorname{BR} p=0.019$, N vs. BR $p=0.033)$.

\section{Discussion}

IOP reduction after XEN-45 implantation in our cohort is comparable with other studies where IOP was lowered from $20.0-25.1 \mathrm{mmHg}$ at baseline to $13.1-17.1 \mathrm{mmHg}$ at 12 months [10-16]. Long-term XEN-45 efficacy data exceeding 12 months of follow-up are still limited. Data at 24 months are available from Gabbay et al. [13] and Reitsamer et al. [16] where they reported an IOP of 14.5-15.2 at $0.5-1.1 \mathrm{GM}$, which is well consistent with our IOP at that timepoint albeit with a higher number of GM in our data.

The needling or bleb revision rate $(49.6 \%)$ was high in our cohort. Depending on different treatment strategies, previous studies reported either more needlings or bleb revisions. While Widder et al. [14] followed a strategy to perform only bleb revisions (revision rate $34.0 \%$ at 8.5 months mean follow-up) and no needlings, the group of Gabbay et al. [13] rather relied on needlings (Needling rate 36.8\% at 24 months). In general, the rate for secondary interventions was between 22.1 and $44.0 \%$ in the studies mentioned above [10-16].

Midha et al. [17] conducted a prospective trial to evaluate needling outcomes in 51 eyes, where IOP was lowered from 23.6 to $14.3 \mathrm{mmHg}$ at an average follow-up time of 17.0 months. As no medication values were given and multiple needlings were allowed, a comparison to our results appears difficult. However, following a needling procedure, in Midhas case series, about $30 \%$ of the cases required reoperation, whereas in our cohort, $53 \%$ required either open bleb revision $(45 \%)$ or a secondary glaucoma surgery $(8 \%)$. The discrepancy in these numbers may be explained by the different approaches towards patients with failed needling. While they performed a second needling in about $40 \%$, we did so in only $4 \%$ and instead relied on the effect of an open bleb revision.

Linton et al. [18] analyzed outcomes of a XEN-45 revision surgery method, similar to our bleb revision strategy, including 16 eyes resulting in an IOP reduction from $26.1 \mathrm{mmHg}$ with $2.0 \mathrm{GM}$ to $16.3 \mathrm{mmHg}$ with $0.7 \mathrm{GM}$ after 1 year of follow-up. A further needling after bleb revision was required in four and a further drainage surgery in three of 16 eyes. In our case series, we performed bleb revisions in 105 eyes, out of which one required bleb needling, seven required a second bleb revision, and ten eyes required secondary glaucoma surgery. Linton et al. report a QS21 of 56\% at 1 year, which is well comparable to our QS21 of $50.7 \%$. It is noteworthy that we reported the crude success of bleb revision not allowing for any further intervention, while in Linton's series, two out of the 9 eyes counted as success at 12 months postoperatively even though they required intermittent bleb needling. Subtracting these two eyes, their QS21 would have been $44 \%$ instead of $56 \%$.

To the best of our knowledge, this is the first study to present real-life data of bleb needling $(\mathrm{N})$ and open bleb revision (BR) after XEN-45 implantation where no further intervention was allowed to get comparable results of the specific procedure. Furthermore, we report results of a group of patients allowing for postoperative needling and 
bleb revision (PSEA) as part of the planned procedure and compare this to the results of pure XEN-45 surgery (PS), not allowing for any further intervention. Kaplan-Meier survival analyses resulted in survival rates being markedly different between the groups. At 1 year postoperatively for the QS21 and the QS18 criteria, the survival was significantly better for the BR group compared to the PS and the $\mathrm{N}$ group while the latter two were not significantly different from each other. The IoGM criterion (IOP reduction or medication reduction) resulted in a lower success rate in the $\mathrm{N}$ group compared to the PS and the BR group while there was no difference between PS and BR group. Together, these findings suggest that in our study cohort, open bleb revision is superior to slit-lamp-based bleb needling and even better than the primary surgery itself. This finding is also supported by the median survival times, which were significantly longer for the BR $(\mathrm{QS} 21=423$ days $)$ compared to the $\mathrm{PS}$ group $(\mathrm{QS} 21=176$ days $)$.

The more favorable results of open bleb revision compared to primary XEN-45 implantation are surprising. Usually, in glaucoma surgery, a surgical revision would not be expected to surpass the primary surgery in terms of efficacy. Due to its retrospective nature, our study cannot be taken as definite proof of the superiority of BR over PS. For further conclusions about this, we should await other confirming evidence. However, our results might be explained by the inherently uncertain position of the external end of the XEN-45 relative to the conjunctiva or Tenon's capsule. This circumstance may trigger a scarring response in some cases which is avoided after open bleb revision because of a clear position of the XEN-45 below the conjunctiva and-if not resected-the Tenon's capsule. Another possible reason for our findings might be the fact that we usually resected the cicatrized Tenon's capsule in the approximately two clock hour positions adjacent to the XEN-45.

No differences in success were observed between standalone XEN-45 implantation and combined XEN-45 surgery with cataract extraction, although the Cox proportional hazards model revealed a lower hazard ratio for failure in the combined group $(\mathrm{HR}=0.55, p=0.01)$. We think that a combination of factors may have caused this finding: a larger proportion of angle-closure glaucoma in the combined cases ( 24.0 vs. $5.7 \%$ ), lower preoperative IOP in the combined cases $(20.7 \pm 6.3$ vs. $24.5 \pm 8.6 \mathrm{mmHg})$, higher failure risk for eyes with lower preoperative IOP, but still comparable success rates.

Since success rates for the subgroup with a preoperative IOP $<21 \mathrm{mmHg}$ showed more unsatisfactory results in the QS21, QS18, and IoGM criteria, our data suggest that XEN45 is a less suitable option, especially in patients with low preoperative IOP values.

Due to the retrospective nature of the study and the open design, we cannot exclude an effect of selection and detection bias. Our cohort was characterized by a large attrition rate where good cases may have been discharged while bad cases remained in our out-patient clinic for regular checkups. Furthermore, our treatment strategy led to IOP values reflecting the preferences of the treating physicians by masking not acceptable IOP values with constant needlings and bleb revisions. As we started to implant the XEN-45, needling was the preferred revision strategy. Later, we got the impression that bleb revision leads to preferable results. This resulted in $24 \%$ of the bleb revisions being preceded by needlings, while only $4 \%$ of the needlings were preceded by a bleb revision. For a more precise analysis, a prospective approach with longer follow-up and sophisticated methods is needed.

\section{Conclusion}

The XEN-45 implant is an effective and safe MIGS device. However, in our study cohort, $49.6 \%$ of eyes needed at least one postoperative secondary intervention. Although previous studies present data on needling and bleb revision outcomes after XEN-45 implantation, no comparison between these two postoperative interventions has been reported. In the present study, our data appear to indicate that open filtering bleb revision might have beneficial outcomes in terms of Kaplan-Meier success rates, and the reintervention rate compared to the needling procedure.

Funding Open access funding provided by Medical University of Vienna.

Data availability On demand.

Code availability Not applicable.

\section{Declarations}

Ethics approval and consent to participate The study was approved by the ethics committee of the Medical University of Vienna.

Since this was a retrospective study, no informed consent was given by the study subjects.

Consent for publication All authors agree with the publication.

Conflict of interest The authors declare no competing interests.

Open Access This article is licensed under a Creative Commons Attribution 4.0 International License, which permits use, sharing, adaptation, distribution and reproduction in any medium or format, as long as you give appropriate credit to the original author(s) and the source, provide a link to the Creative Commons licence, and indicate if changes were made. The images or other third party material in this article are included in the article's Creative Commons licence, unless indicated 
otherwise in a credit line to the material. If material is not included in the article's Creative Commons licence and your intended use is not permitted by statutory regulation or exceeds the permitted use, you will need to obtain permission directly from the copyright holder. To view a copy of this licence, visit http://creativecommons.org/licenses/by/4.0/.

\section{References}

1. Kirwan JF, Lockwood AJ, Shah P, Macleod A, Broadway DC, King AJ et al (2013) Trabeculectomy in the 21st century: a multicenter analysis. Ophthalmology 120(12):2532-2539

2. Arriola-Villalobos P, Martínez-de-la-Casa JM, Díaz-Valle D, Fernández-Pérez C, García-ánchez J, García-Feijoó J (2012) Combined iStent trabecular micro-bypass stent implantation and phacoemulsification for coexistent open-angle glaucoma and cataract: a long-term study. Br J Ophthalmol 96(5):645-649

3. Gonnermann J, Bertelmann E, Pahlitzsch M, Maier-Wenzel AKB, Torun N, Klamann MKJ (2017) Contralateral eye comparison study in MICS \& MIGS: Trabectome ${ }^{\circledR}$ vs. iStent inject ${ }^{\circledR}$. Graefe's Arch Clin Exp Ophthalmol 255(2):359-65

4. Ahmed IIK, Fea A, Au L, Ang RE, Harasymowycz P, Jampel HD et al (2020) A prospective randomized trial comparing Hydrus and iStentmicroinvasive glaucoma surgery implants for standalone treatment of open-angle glaucoma: the COMPARE study. Ophthalmology 127(1):52-61

5. Kaplowitz K, Bussel II, Honkanen R, Schuman JS, Loewen NA (2016) Review and meta-analysis of ab-interno trabeculectomy outcomes. Br J Ophthalmol 100(5):594-600

6. Lin ZJ, Xu S, Huang SY, Bin ZX, Zhong YS (2016) Comparison of canaloplasty and trabeculectomy for open angle glaucoma: a meta-analysis. Int J Ophthalmol 9(12):1814-1819

7. Reiss G, Clifford B, Vold S, He J, Hamilton C, Dickerson J et al (2019) Safety and effectiveness of CyPasssupraciliary micro-stent in primary open-angle glaucoma: 5-year results from the COMPASS XT study. Am J Ophthalmol. 208:219-25. https://doi.org/ 10.1016/j.ajo.2019.07.015

8. Salinas L, Chaudhary A, Berdahl JP, Lazcano-Gomez GS, Williamson BK, Dorairaj SK et al (2018) Goniotomy using the Kahook Dual Blade in severe and refractory glaucoma: 6-month outcomes. J Glaucoma 27(10):849-855
9. Lavia C, Dallorto L, Maule M, Ceccarelli M, Fea AM (2017) Minimally-invasive glaucoma surgeries (MIGS) for open angle glaucoma: a systematic review and meta-analysis. PLoS ONE 12:1-33

10. Mansouri K, Gillmann K, Rao HL, Guidotti J, Mermoud A (2018) Prospective evaluation of XEN gel implant in eyes with pseudoexfoliative glaucoma. J Glaucoma 27(10):869-873

11. Gillmann K, Bravetti GE, Mermoud A, Rao HL, Mansouri K (2019) XEN gel stent in pseudoexfoliative glaucoma: 2-year results of a prospective evaluation. J Glaucoma 28(8):676-684

12. Heidinger A, Schwab C, Lindner E, Riedl R, Mossböck G (2019) A retrospective study of 199 Xen 45 stent implantations from 2014 to 2016. J Glaucoma 28(1):75-79

13. Gabbay IE, Allen F, Morley C, Pearsall T, Bowes OM, Ruben S (2019) Efficacy and safety data for the XEN45 implant at 2 years: a retrospective analysis. Br J Ophthalmol 104(8):1125-1130

14. Widder RA, Dietlein TS, Dinslage S, Kühnrich P, Rennings C, Rössler G (2018) The XEN45 Gel Stent as a minimally invasive procedure in glaucoma surgery: success rates, risk profile, and rates of re-surgery after 261 surgeries. Graefe's Arch Clin Exp Ophthalmol 256(4):765-771

15. Smith M, Charles R, Abdel-Hay A, Shah B, Byles D, Lim LA et al (2019) 1-year outcomes of the Xen45 glaucoma implant. Eye 33(5):761-766

16. Reitsamer H, Sng C, Vera V, Lenzhofer M, Barton K, Stalmans I et al (2019) Two-year results of a multicenter study of the ab interno gelatin implant in medically uncontrolled primary open-angle glaucoma. Graefe's Arch Clin Exp Ophthalmol 257(5):983-996

17. Midha N, Gillmann K, Chaudhary A, Mermoud A, Mansouri K (2020) Efficacy of needling revision after XEN gel stent implantation: a prospective study. J Glaucoma 29(1):11-14

18. Linton E, Au L (2020) Technique of Xen implant revision surgery and the surgical outcomes: a retrospective interventional case series. Ophthalmol Ther 9(1):149-157

Publisher's note Springer Nature remains neutral with regard to jurisdictional claims in published maps and institutional affiliations. 